\title{
Photo-Crosslinking Analysis of Preferential Interactions between a Transmembrane Peptide and Matching Lipids ${ }^{\dagger}$
}

\begin{abstract}
Anja N. J. A. Ridder, ${ }^{*}$, Robin E. J. Spelbrink, ${ }^{\ddagger}$ Jeroen A. A. Demmers, ${ }^{\S}$ Dirk T. S. Rijkers," Rob M. J. Liskamp," Josef Brunner, ${ }^{\perp}$ Albert J. R. Heck, ${ }^{\S}$ Ben de Kruijff, ${ }^{\ddagger}$ and J. Antoinette Killian ${ }^{\ddagger}$

Department of Biochemistry of Membranes, Center for Biomembranes and Lipid Enzymology, Institute of Biomembranes, Utrecht University, Padualaan 8, 3584 CH Utrecht, The Netherlands, Department of Biomolecular Mass Spectrometry, Bijvoet Center for Biomolecular Research and Utrecht Institute for Pharmaceutical Sciences, Utrecht University, Sorbonnelaan 16, 3584 CA Utrecht, The Netherlands, Department of Medicinal Chemistry, Utrecht Institute for Pharmaceutical Sciences, Faculty of Pharmaceutical Sciences, Utrecht University, Sorbonnelaan 16, 3584 CA Utrecht, The Netherlands, and Institute of Biochemistry, Swiss Federal Institute of Technology Zürich (ETH), Hönggerberg, CH-8093 Zürich, Switzerland
\end{abstract}

Received January 13, 2004; Revised Manuscript Received January 30, 2004

\begin{abstract}
In this study, a novel method is presented by which the molecular environment of a transmembrane peptide can be investigated directly. This was achieved by incorporating a photoactivatable crosslinking probe in the hydrophobic segment of a model transmembrane peptide. When this peptide was incorporated into lipid bilayers and irradiated with UV light, a covalent bond was formed between the crosslinking probe and a lipid. This crosslinking reaction could be visualized by sodium dodecyl sulfate-polyacrylamide gel electrophoresis and the resulting product could be characterized by mass spectrometry. By use of phospholipases, it was demonstrated that the peptide crosslinks to both acyl chains of the lipids. The peptide showed a clear preference to partition into fluid lipids and was excluded from lipids in the gel phase. However, when the peptide was incorporated into bilayers containing two lipid species with different acyl chain lengths, molecular sorting of the lipids around the peptide based on hydrophobic matching was not observed. It is proposed that the size of the transmembrane part plays an important role in the dynamic interactions of membrane proteins with the surrounding lipids and hence in determining whether molecular sorting can occur.
\end{abstract}

Proteins in biological membranes reside in a complex environment in which they are surrounded by many different lipid species. Transmembrane proteins can have different affinities for different lipids, and as a result particular lipids or members of particular lipid classes may become enriched around a certain protein. Such differences in affinity may be due to specific interactions with the lipid headgroups or the lipid acyl chains. An example of the latter would be the enrichment of matching lipids around a protein, which could be a mechanism by which mismatches between hydrophobic transmembrane segments and the hydrophobic thickness of the lipid bilayer can be relieved. Such a mechanism of molecular sorting by transmembrane proteins has been

$\dagger$ This work was supported by the Chemical Sciences Division (CW) of the Dutch Organization for Scientific Research (NWO).

* Corresponding author: present address Chemie der Biopolymere, Technical University of Munich, Weihenstephaner Berg 3, 85354 Freising, Germany; fax +49 8161 714404; tel +49 8161 713853; e-mail A.Ridder@lrz.tum.de.

$\lessgtr$ Department of Biochemistry of Membranes, Center for Biomembranes and Lipid Enzymology, Institute of Biomembranes, Utrecht University.

${ }^{\S}$ Department of Biomolecular Mass Spectrometry, Bijvoet Center for Biomolecular Research and Utrecht Institute for Pharmaceutical Sciences, Utrecht University.

" Department of Medicinal Chemistry, Utrecht Institute for Pharmaceutical Sciences, Faculty of Pharmaceutical Sciences, Utrecht University.

$\perp$ Swiss Federal Institute of Technology. predicted from theoretical calculations $(1,2$; reviewed in refs 3 and 4 ) and has indeed been shown to occur for several transmembrane proteins $(5-8)$.

To gain insight into the role of protein-lipid interactions in the sorting of lipids around specific proteins, tools must be available to determine the lipid environment of membrane proteins. Previously, this has been probed mainly by fluorescence and $\mathrm{ESR}^{1}$ methods using labeled lipids $(5-11)$. In this study, we explore a more direct method to investigate the immediate surroundings of membrane proteins. For this purpose we make use of a synthetic model peptide, which contains a photoactivatable probe that is able to form covalent bonds with the surrounding lipids upon UV irradiation. The crosslinking reaction can be visualized by SDS-PAGE analysis and the identity of the lipid that is crosslinked to the peptide can subsequently be characterized by mass spectrometry.

\footnotetext{
${ }^{1}$ Abbreviations: DCM, dichloromethane; DIPEA, $N, N$-diisopropylethylamine; ESI-MS, electrospray ionization mass spectrometry; ESR, electron spin resonance; Fmoc-Phe(Tmd)-OH, L- $N$-[[[fluorenylmethyl]oxy]carbonyl]-4'-[3-(trifluoromethyl-3H-diazirin-3-yl)]phenylalanine; HATU, $N$-[(dimethylamino)-1H-1,2,3-triazolo[4,5-bipyridin-1-ylmethylene]- $N$-methylmethanaminium hexafluorophosphate; HOAt, $N$-hydroxy-7-azabenzotriazole; HPLC, high-performance liquid chromatography; MLVs, multilamellar vesicles; NMP, $N$-methylpyrrolidone; PC, phosphatidylcholine; SDS-PAGE, sodium dodecyl sulfate-polyacrylamide gel electrophoresis; TFA, trifluoroacetic acid; TFE, trifluoroethanol; TMD-phenylalanine, 3-(trifluoromethyl-3H-diazirin-3-yl)phenylalanine.
} 
A
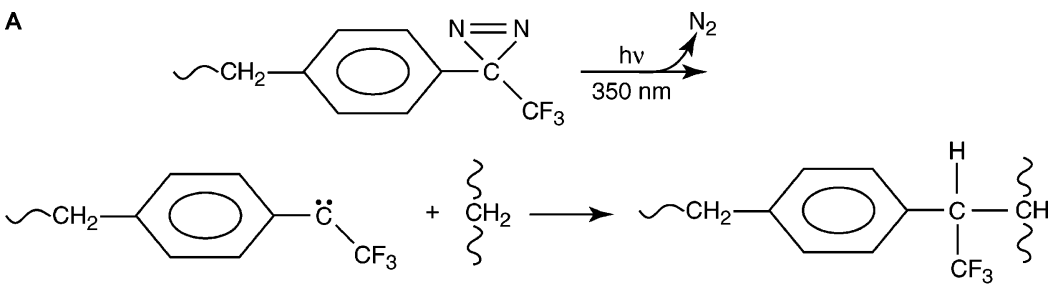

B

WALP23-TMD6

Ac-GWWLAF*ALALALALALALALWWA-Am

WALP23-TMD8

Ac- GWWLALAF*ALALALALALALWWA-Am

FIGURE 1: (A) Photo-crosslinking reaction. The TMD-phenylalanine side chain is shown, which upon reaction with UV light loses a nitrogen molecule, resulting in the formation of a reactive carbene. The reaction of such a carbene with a $\mathrm{C}-\mathrm{H}$ bond is shown (adapted from ref 22). (B) Sequence of transmembrane peptides used in this study. The photoactivatable residue is shown in boldface type. Ac, acetyl; Am, amide; F*, TMD-phenylalanine.

As a model transmembrane peptide we chose the WALP23 peptide (see Figure 1), which mimics the transmembrane segment of membrane proteins. This peptide, with a total length of 23 amino acids, consists of a hydrophobic sequence of alternating alanine and leucine residues and is flanked on both sides by two tryptophans. WALP-peptides form stable transmembrane $\alpha$-helices and have been used extensively to study various aspects of peptide/lipid-interactions (1217). A WALP-peptide containing a photoactivatable probe was synthesized and used to investigate preferential sorting based on hydrophobic matching in mixed lipid bilayers. The results provide new insights into mismatch-dependent molecular sorting and illustrate the potential of this crosslinking approach to investigate the nearest neighbors of proteins in complex lipid environments.

\section{EXPERIMENTAL PROCEDURES}

Materials. All lipids were obtained from Avanti Polar Lipids. Bee venom phospholipase $\mathrm{A}_{2}$ and Bacillus cereus phospholipase $\mathrm{C}$ were obtained from Sigma. The photocrosslinking probe Tmd-Phe was incorporated into a WALP23peptide at either the 6 (WALP-TMD6) or 8 (WALP-TMD8) position during chemical synthesis as follows. Synthesis of the peptide was performed on an Applied Biosystems 433A peptide synthesizer using the FastMoc protocol on a $0.25 \mathrm{mmol}$ scale, as described previously (18) until the position of the photolabel was reached. Then, L- $N$-[[[fluorenylmethyl]oxy]carbonyl]-4'-[3-(trifluoromethyl-3H-diazirin3-yl)]phenylalanine (Fmoc-Phe(Tmd)-OH) was coupled, in an excess of only 2 equiv, with $N$-[(dimethylamino)$1 H$-1,2,3-triazolo[4,5-bipyridin-1-ylmethylene]- $N$-methylmethanaminium hexafluorophosphate/ $N$-hydroxy-7-azabenzotriazole (HATU/HOAt), in the presence of 4 equiv of $N, N$ diisopropylethylamine (DIPEA) in $N$-methylpyrrolidone (NMP) for $16 \mathrm{~h}$. The remaining amino acid sequence was synthesized manually. Acetylation of the amino functionality of the final amino acid (glycine) was performed with acetic anhydride/ DIPEA/HOAt in NMP during 15 min followed by washing with NMP and DCM, after which the peptide-resin was dried in a vacuum desiccator. The peptide was cleaved from the resin and deprotected in TFA/1,2-ethanedithiol/ $\mathrm{H}_{2} \mathrm{O}$ 95: 2.5:2.5. Peptide purity was analyzed by analytical HPLC and electrospray ionization mass spectrometry (ESI-MS) and was determined to be $95 \%$ or higher.

Peptide Incorporation into Lipid Vesicles. Peptides were incorporated into lipid vesicles essentially as described (14).
Briefly, peptide that was dissolved in TFE $(1 \mathrm{mg} / \mathrm{mL})$ was mixed with lipids in either chloroform/methanol $(1: 1)$ or chloroform alone at the desired peptide/lipid molar ratio (ranging from 1:25 to 1:100). The solvent was removed by evaporation under a stream of nitrogen and the peptide/lipid film was further dried overnight under vacuum. The mixed films were hydrated with $0.5 \mathrm{~mL}$ of $20 \mathrm{mM}$ ammonium acetate, $\mathrm{pH} 7.5$, and dispersed by vigorous vortexing, resulting in a lipid concentration of $1.88 \mathrm{mM}$. Hydration was performed at room temperature or, when lipids with saturated acyl chains were present, at $60{ }^{\circ} \mathrm{C}$, and the samples were subjected to three cycles of freeze-thawing. The resulting multilamellar vesicles (MLVs) were stored at $4{ }^{\circ} \mathrm{C}$ until use. These procedures were carried out in the dark as much as possible.

Photo-crosslinking. Photo-crosslinking was performed in thin-walled $0.2 \mathrm{~mL}$ SoftTubes (Biozym). Generally, $20 \mu \mathrm{L}$ of MLVs with incorporated peptides were irradiated with a $15 \mathrm{~W}$ UV light source (CAMAC universal lamp) at $366 \mathrm{~nm}$. When crosslinking was performed at room temperature, the tube with the MLVs was laid directly on top of the lamp. When crosslinking was performed at $0{ }^{\circ} \mathrm{C}$, the tubes were placed on ice and the lamp was placed on top of the opened tubes. When crosslinking was performed at elevated temperatures, the tubes were placed in a thermoincubator and also in this case the lamp was placed on top of the opened tubes.

Phospholipase Treatments. For the phospholipase treatments of crosslinked samples, $0.2 \mathrm{mM}$ di-16:1-PC MLVs with incorporated WALP-TMD6 (1:25) were irradiated for 10 min with UV light and subsequently incubated in $50 \mathrm{mM}$ Tris-HCl buffer ( $\mathrm{pH}$ 7.5) containing 0.4 units of phospholipase and either $1 \mathrm{mM} \mathrm{ZnCl} 2$ (phospholipase $\mathrm{C}$ treatment) or $5 \mathrm{mM} \mathrm{CaCl}_{2}$ (phospholipase $\mathrm{A}_{2}$ treatment). The samples were incubated for $3 \mathrm{~h}$ under shaking, either at $37{ }^{\circ} \mathrm{C}$ (phospholipase $\mathrm{C}$ ) or at room temperature (phospholipase $\mathrm{A}_{2}$ ). Subsequently the peptides and lipids were extracted (19) and after evaporation of the chloroform were taken up in 20 $\mu \mathrm{L}$ of TFE/acetonitrile (2:1) containing $1 \%$ formic acid. For nanoflow ESI-MS measurements these samples were diluted 15-fold and sprayed with the same solvent.

Gel Electrophoresis and Staining. Samples were run on tricine $-16.5 \%$ tricine - SDS - polyacrylamide gels (20). The gels were stained with an ammoniacal silver staining procedure (21). Subsequently the gels were stained with Bio- 

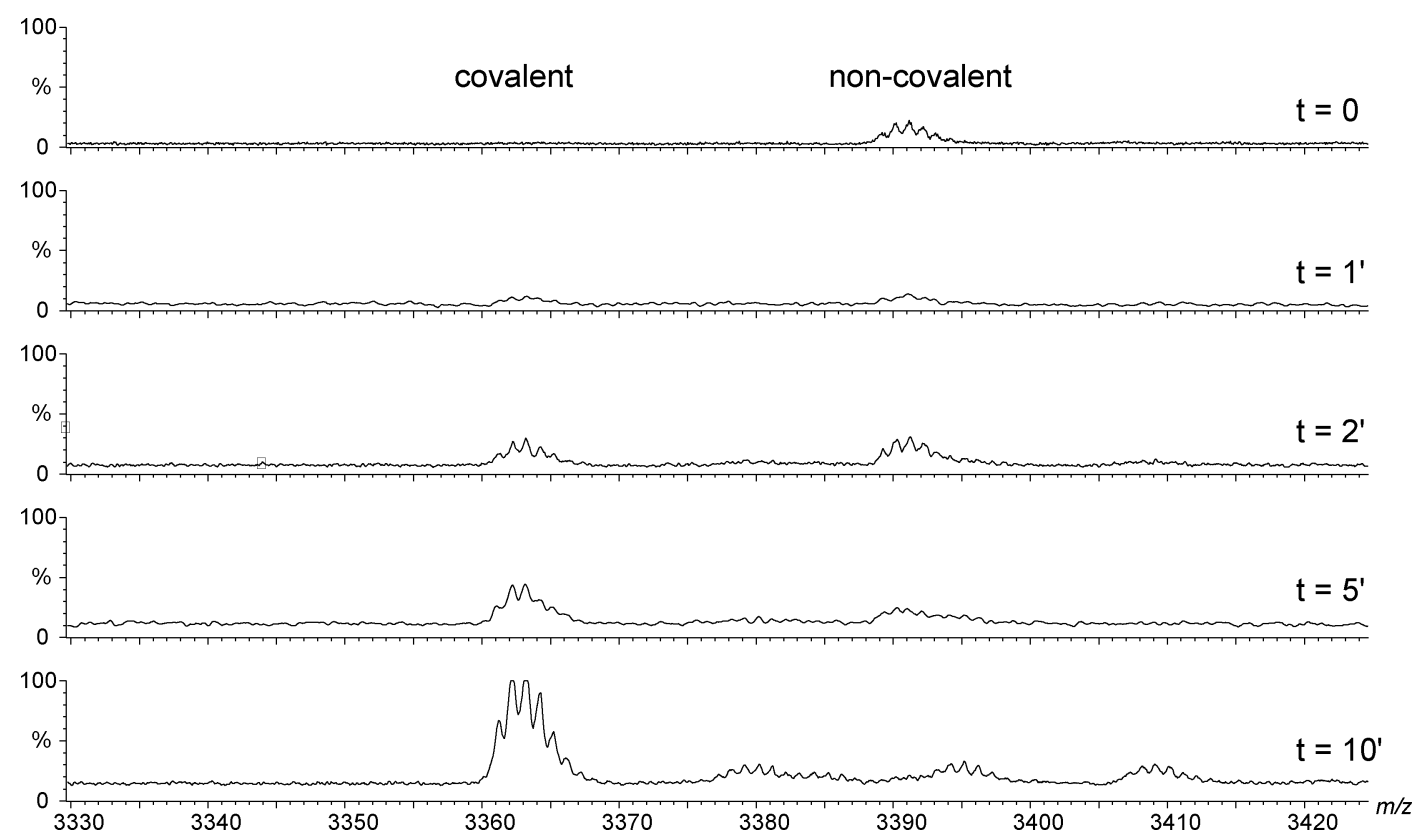

FIGURE 2: Time dependency of crosslinking. Zoom-in ESI-MS spectra of WALP-TMD6 reconstituted into di-16:1-PC in a 1:100 peptide/ lipid ratio and irradiated with UV light for the indicated time periods. The data are normalized with respect to the intensity of the uncrosslinked peptide $(\mathrm{m} / \mathrm{z}, 2661)$. The position of the covalent and noncovalent peptide-lipid complexes are indicated.

Safe Coomassie (Bio-Rad) according to the manufacturer's instructions.

Mass Spectrometric Measurements. Nanoflow ESI-MS measurements were performed on a time-of-flight instrument (Q-Tof or LCT; Micromass Ltd.) operating in positive-ion mode and equipped with a Z-spray nano-ESI source. NanoESI capillaries were prepared as described (14). The nanoESI capillary was positioned approximately $5 \mathrm{~mm}$ before the orifice of the mass spectrometer. The potential between the nano-ESI capillary and the orifice of the mass spectrometer was typically set to $1.8 \mathrm{kV}$, and the cone voltage to 80 $\mathrm{V}$. The source temperature was $80^{\circ} \mathrm{C}$. In MS/MS mode, the quadropole was used to select precursor ions, which were fragmented in the hexapole collision cell, generating product ions that were subsequently mass-analyzed by the orthogonal time-of-flight mass analyzer. Argon was used as collision gas. All samples were sprayed from TFE/acetonitrile (2:1) containing $1 \%$ formic acid.

\section{RESULTS}

A photoactivatable TMD-phenylalanine was incorporated into a WALP23 peptide during its chemical synthesis. Upon activation by UV light of around $360 \mathrm{~nm}$, molecular nitrogen is eliminated from this probe and a very reactive carbene intermediate is generated, which is able to insert even into chemically virtually inert $\mathrm{C}-\mathrm{H}$ bonds (Figure $1 \mathrm{~A} ; 22,23$ ). The TMD-Phe probe was incorporated at position 6 or 8 in a WALP23 peptide, resulting in WALP-TMD6 or WALPTMD8 (Figure 1B).

Detection of Crosslinks to Lipids. To test whether these peptides can indeed crosslink to their lipid surroundings, WALP-TMD6 was incorporated into multilamellar vesicles (MLVs) of di-16:1-PC in a peptide/lipid molar ratio of 1:100. After irradiation with UV light for different time periods, crosslinked products were characterized by ESI-MS (Figure 2). The isotope envelope corresponding to the protonated peptide ion peak of WALP-TMD6 was clearly visible in the spectrum of the non irradiated sample with the expected monotopic $\mathrm{m} / \mathrm{z}$ value of $2661.5 \mathrm{Da}$ (not shown). Moreover, as shown in Figure 2, an isotope envelope with relatively low intensity was detected with an $\mathrm{m} / \mathrm{z}$ value of $3391 \mathrm{Da}$ corresponding to a WALP-TMD6 peptide noncovalently bound to one di-16:1-PC molecule (729.6 Da higher). Upon UV irradiation, a new isotope envelope appeared with an $\mathrm{m} / \mathrm{z}$ ratio that was exactly $28 \mathrm{Da}$ lower $(3363 \mathrm{Da})$. This mass difference corresponds to the loss of one nitrogen molecule, which is eliminated from the peptide after activation of the crosslinking probe, suggesting that these peaks result from the crosslinking reaction between the peptide and a lipid molecule. Upon increasing the time of UV irradiation, the intensity of the peaks corresponding to the covalently crosslinked product gradually increased, whereas that of the noncovalently bound complex was more variable.

In principle it might be possible that the isotope envelope at $3363 \mathrm{Da}$, assigned to a covalent lipid-peptide complex, corresponds to photolyzed peptide that is noncovalently bound to lipid. However, this possibility could be excluded on the basis of two experimental observations. The first observation comes from tandem mass spectrometry (MS/ MS) experiments. WALP-TMD6 was incorporated into diC18:1-PC (1:50) and UV irradiated for $5 \mathrm{~min}$, and the isotope envelope at $3419 \mathrm{Da}$, most likely corresponding to a covalent peptide-lipid complex $[\mathrm{m} / \mathrm{z} 2661-28+786$ (the mass of di-18:1-PC))] was fragmented by collision (Figure 3 ). The spectrum predominantly shows B fragment ions, corresponding to $\mathrm{N}$-terminal fragments of the peptide. Importantly, the observed fragment masses show that the lipid is specifically attached to the peptide at the site of the TMD-Phe probe, since fragment ions can be observed that correspond to the loss of TMD-Phe (minus molecular nitrogen) together with di-18:1-PC but not to the loss of lipid alone. This would be highly unlikely for a noncovalent complex, because even covalent bonds in the peptide are broken under these harsh conditions. The second observation is that the complex stays 




FIGURE 3: MS/MS spectrum of the covalent peptide-lipid complex of WALP-TMD6 and di-18:1-PC. WALP-TMD6 was reconstituted into di-18:1-PC in a 1:50 peptide/ lipid ratio and irradiated with UV light for $5 \mathrm{~min}$. The isotope envelope at $\mathrm{m} / z, 3419$ was fragmented by collision in the second quadrupole. The amino acids that are successively lost from the C-terminus of the peptide are indicated between the resulting B fragment ions, which correspond to N-terminal peptide fragments. Peaks corresponding to different amino acid length peptides are clearly observed from the C-terminus of the peptide up to Ala7. The mass difference with the next observed B fragment corresponds to TMD-Phe with di-18:1-PC but without the molecular nitrogen. Two peaks corresponding to lipids are also indicated.

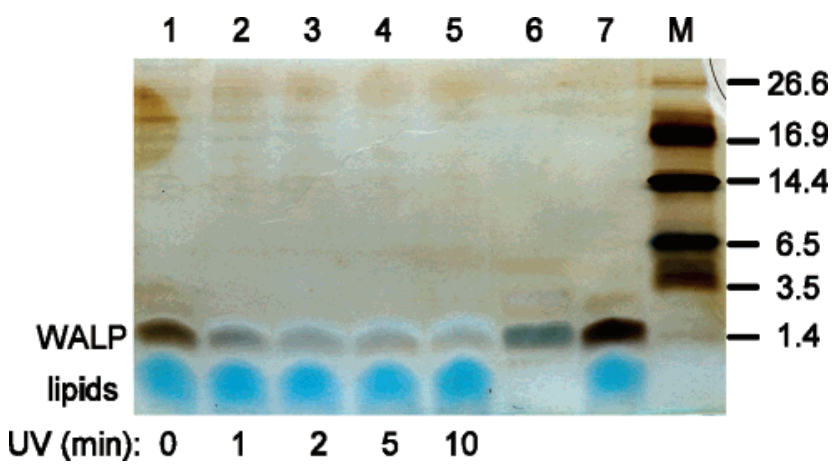

FIGURE 4: Time dependency of crosslinking as analyzed by SDSPAGE. Samples were run on an SDS-Tricine gel, which was stained with silver and subsequently with Coomassie Brilliant Blue. Lanes 1-5 contain the same samples as indicated in Figure 2. Lane 6 contains the stock solution of WALP-TMD6 in TFE, and lane 7 contains WALP23 (without a photo-crosslinking probe) in the same lipids at a 1/100 peptide/lipid ratio. The molecular masses of the marker proteins ( $\mathrm{M}$, in kilodaltons) are indicated.

intact during SDS-polyacrylamide gel electrophoresis (see below), under which conditions noncovalent lipid/protein complexes are normally dissociated. Thus it can be concluded that the WALP-TMD6 peptide is indeed able to crosslink to the lipids surrounding it. The same could be concluded for WALP-TMD8 (data not shown).

The crosslinking reaction was also monitored by SDSPAGE with an SDS - tricine gel (Figure 4). The gel was first stained with silver and subsequently with Coomassie blue. The WALP-TMD6 peptide stains with silver (lane 6) and runs at the same position as the unmodified WALP23 (lane 7). In lane 7, the peptide is incorporated in lipid vesicles. Upon staining with Coomassie blue, these lipids are visible as an intense blue band with a lower molecular weight. Upon
UV irradiation of the vesicles, the intensity of the WALP band decreases and a small blue-colored band appears above it (lanes 1-5). The position of this band corresponds to a slightly higher molecular weight than that of the WALP peptides and its intensity increases with longer irradiation times. Therefore, and because of its blue coloring, it is most likely that this band represents the product of the crosslinking reaction, i.e., WALP-TMD6 covalently attached to a lipid. The apparent decrease in intensity of the total amount of peptide upon UV irradiation is attributed to the differences in staining properties of the peptide and its crosslinked product. Thus, it can be concluded that the crosslinking reaction can be monitored by SDS-PAGE as well as by mass spectrometry.

To establish to which part of the lipid the peptide crosslinks, we made use of phospholipases to cleave the phospholipid and we investigated the reaction products by ESI-MS (24). The WALP-TMD6 peptide was reconstituted in di-16:1-PC, crosslinking was performed, and samples were subsequently treated with phospholipases $\mathrm{A}_{2}$ or C. Phospholipase $\mathrm{C}$ cleaves off the phosphorylcholine headgroup, yielding an isotope envelope with an average $\mathrm{m} / \mathrm{z}$ of 3198 , corresponding to the peptide crosslinked to a lipid without its headgroup. Peaks representing the peptide crosslinked to a phosphorylcholine moiety (expected $m / z$ 2816) were not observed. Phospholipase $\mathrm{A}_{2}$ cleaves off the fatty acid at the sn-2 position of the phospholipid, yielding an isotope envelope with an average $\mathrm{m} / \mathrm{z}$ of 2886 , corresponding to the peptide crosslinked to a palmitoyl chain and with an average at $\mathrm{m} / \mathrm{z} 3125$, corresponding to the peptide connected to a lyso-PC molecule (data not shown). These results demonstrate that the peptide crosslinks to both acyl chains of a phospholipid. 
A
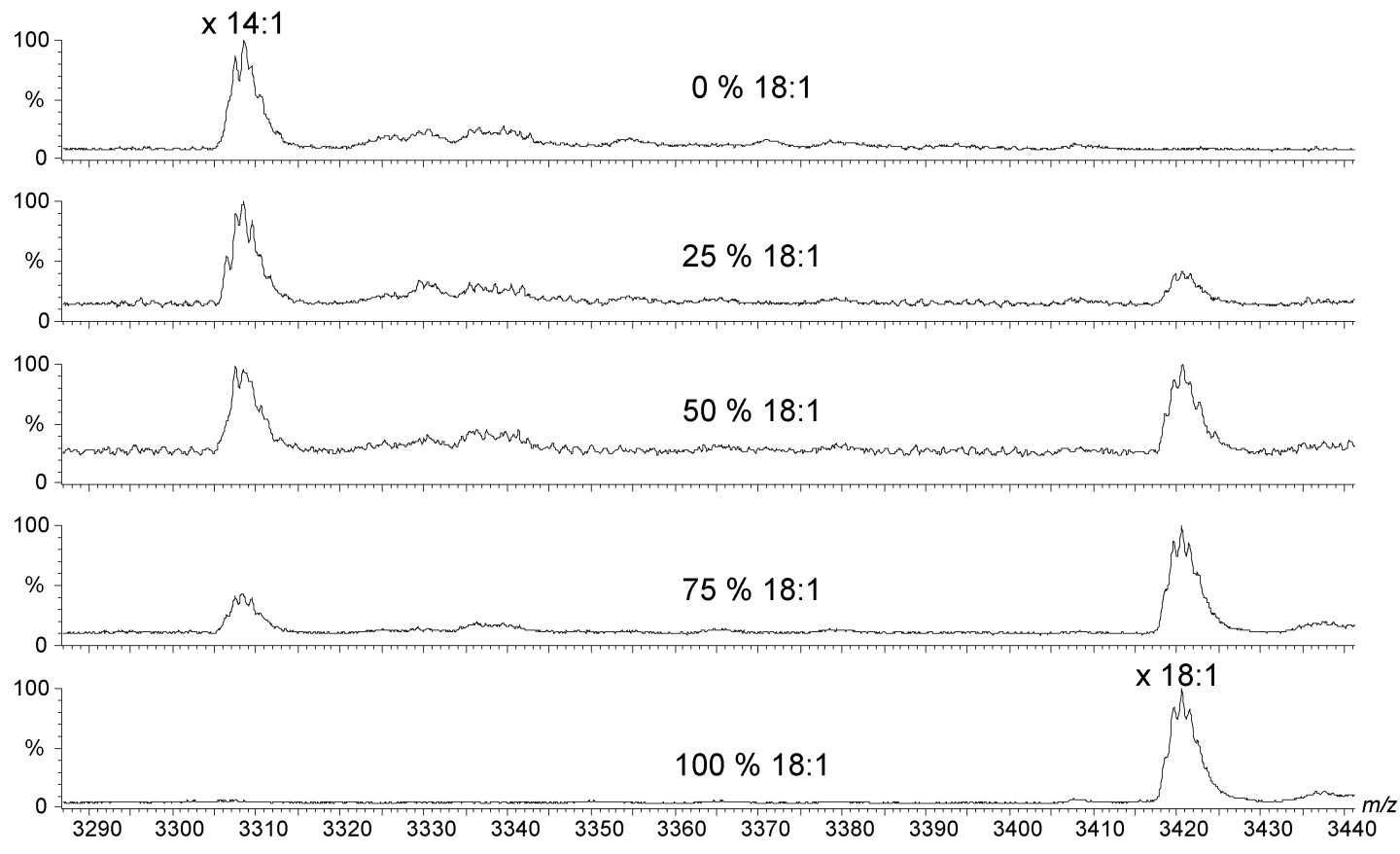

B

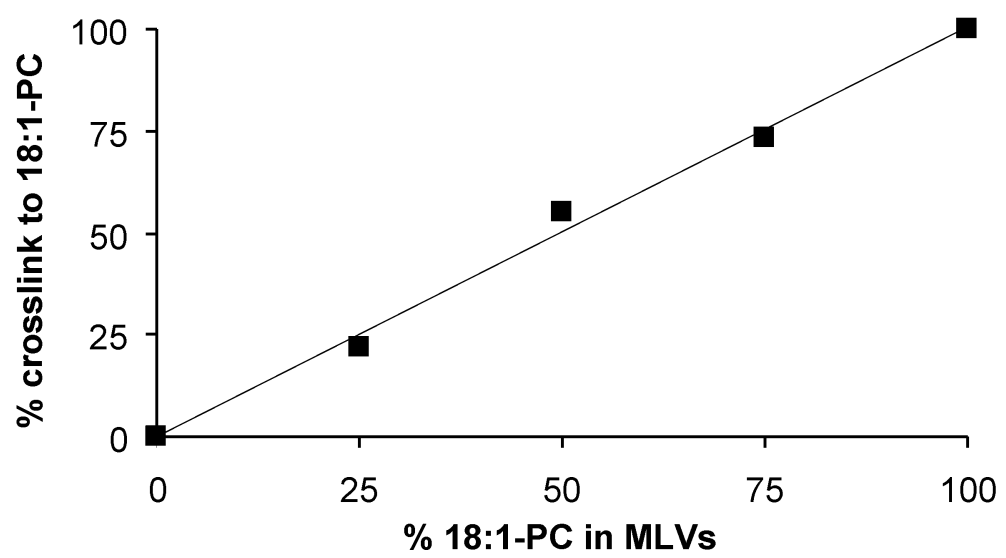

FIGURE 5: (A) ESI-MS spectra of mixtures of di-14:1-PC and di-18:1-PC after crosslinking. WALP-TMD6 was reconstituted in a 1:25 peptide/lipid ratio into MLVs with the indicated amount of di-18:1-PC andirradiated with UV light for 10 min. The positions of the crosslinked products are indicated. (B) Comparison between the theoretical amount of di-18:1-PC in the MLVs and the percentage of crosslink to di-18:1-PC (quantified as the area of the peaks in the MS spectra and expressed as percent of the total amount of crosslinks to both di-14:1-PC and di-18:1-PC).

Crosslinking to Lipids with Different Acyl Chain Lengths. To investigate whether the WALP-TMD peptide displays preferential interactions with certain lipids due to hydrophobic matching, MLVs composed of more than one lipid species were used. First, WALP-TMD6 was reconstituted in MLVs containing mixtures of di-14:1-PC and di-18:1PC. Judged from previous results, the peptide is expected to match best with di-18:1-PC (25) and thus might preferentially accumulate this lipid around itself. Crosslinking was performed at room temperature and the results are shown in Figure 5. In the spectra, crosslink peaks to di-14:1-PC and di-18:1-PC are observed $(\mathrm{m} / \mathrm{z} 3306$ and 3418, respectively) (Figure 5A). The relative intensity of these crosslinked peaks corresponds to the relative amount of each lipid in the vesicles (Figure 5B). Thus, we conclude that the peptide does not exhibit a preferential interaction with either di-14:1-PC or di-18:1-PC. Very similar results were obtained in mixtures of di-16:1-PC and di-20:1-PC (not shown).
Next, WALP-TMD6 was incorporated into MLVs (1:25 peptide/lipid molar ratio) composed of equal amounts of the saturated lipids di-12:0-PC and di-18:0-PC. The lipids in this system have a slightly larger length difference than those used for the previous experiments, and in addition they can undergo phase separation, depending on the temperature. The crosslinking reaction was therefore performed at different temperatures. At $0{ }^{\circ} \mathrm{C}$ and at room temperature, the two lipids are expected to phase-separate, with di-12:0-PC being in the fluid phase and di-18:0-PC in the gel phase (2). When crosslinking was performed at $0{ }^{\circ} \mathrm{C}$, virtually only crosslinked products of the peptide with di-12:0-PC could be detected in the spectrum at $m / z 3254$ (Figure 6). At room temperature, some crosslinking of the peptide to di-18:0-PC was detected $(\mathrm{m} / \mathrm{z}, 3422)$. At $60{ }^{\circ} \mathrm{C}$, at which temperature both lipids are in the fluid phase and are expected to mix completely, the crosslinking efficiency to di-12:0-PC and di-18:0-PC was found to be approximately equal. At this temperature also a 



FIGURE 6: ESI-MS spectra of a 1:1 mixture of di-12:0-PC and di-18:0-PC after crosslinking at different temperatures. WALP-TMD6 was reconstituted in a 1:25 peptide/lipid ratio into MLVs containing equal amounts of di-12:0-PC and di-18:0-PC and irradiated with UV light for $10 \mathrm{~min}$ at the indicated temperatures. The positions of the crosslinked products are indicated. In these spectra, noncovalent peptidelipid complexes can also be observed.

significant amount of noncovalent lipid-peptide complexes is observed, again both of approximately equal intensity for di-C12:0-PC and di-C18:0-PC. The reason for their increased appearance at this temperature is not clear. In any case, the results indicate that at $0{ }^{\circ} \mathrm{C}$ the WALP-TMD6 peptide prefers to reside in the fluid phase of the bilayer, where it is likely to be completely surrounded by di-12:0-PC. At room temperature, some di-18:0-PC molecules may be mixed with the di-12:0-PC in the fluid phase, while at $60^{\circ} \mathrm{C}$ the peptide crosslinks to both lipids approximately to the same extent, despite their length difference. Similar results were observed with the WALP-TMD8 peptide (not shown), where the probe is located slightly deeper into the hydrophobic interior of the membrane and at the opposite face of the helix. Thus these results are in agreement with the finding from the previous experiments that the peptide does not preferentially surrounds itself with matching lipids.

\section{DISCUSSION}

In this study, a new approach is presented to investigate the lipid surroundings of a transmembrane peptide directly from the peptide's point of view. This was accomplished by incorporating the photoactivatable crosslinker TMDphenylalanine into a model transmembrane peptide. The model peptide that was used in this study was WALP23, which has been used extensively in the study of proteinlipid interactions $(12-17)$. However, the method would be suitable for any synthetic transmembrane peptide, and even for larger membrane proteins, by incorporating the probe either by chemical modification of a reactive amino acid side chain (24) or by using in vitro translation systems (26). Upon activation with UV light, a highly reactive carbene intermediate is generated with a lifetime within the nanosecond range, which is able to crosslink even to chemically inert $\mathrm{C}-\mathrm{H}$ bonds and thus to lipid acyl chains $(22,27)$. Crosslinking of the probe in WALP-TMD to lipids could be visualized by SDS-PAGE, and it was possible to identify the lipids crosslinked to the peptide by ESI-MS. By use of phospholipases, it could be demonstrated that crosslinking takes place with the lipid acyl chains, as would be expected for a peptide in a transmembrane orientation.

The results obtained for bilayers composed of mixtures of saturated lipids directly demonstrate the ability of the crosslinking reaction to probe the molecular environment of the peptide. In the mixture of di-12:0-PC and di-18:0PC, phase separation occurs within the bilayer between disordered di-12:0-PC and ordered di-18:0-PC at low temperatures. Under these conditions crosslinks are observed only with di-12:0-PC. This is not due to an inability of the peptide to crosslink with ordered lipids, since crosslinks are observed when the peptide is incorporated in lipid bilayers of pure disaturated PC at gel-state conditions (not shown). Thus, the results demonstrate that WALP-TMD is preferentially surrounded by di-12:0-PC in a mixture with di-C18:0-PC at low temperature and hence is excluded from the gel-state di-18:0-PC domains. This has also been observed for other transmembrane peptides as well as larger proteins $(2,28,29)$ and is due to the tight packing of lipids in the gel state. At higher temperatures where both lipids are in the fluid phase, WALP-TMD crosslinks to both lipids with approximately equal efficiency despite their large difference in chain length. This unambiguously demonstrates that no molecular sorting takes place around the peptide, although it has a length that closely matches that of fluid di-18:0-PC (25). This conclusion is further substantiated by the results of the mixtures of the unsaturated PC species. Also in these systems no molecular sorting takes place under 
mismatching conditions in either di-14:1-PC/di-18:1-PC or di-16:1-PC/di-20:1-PC mixtures.

Literature data so far have shown molecular sorting of lipids to occur to various extents for a number of membrane proteins $(5-8)$. Then why do we not observe it in our systems? We suggest that the explanation lies in the large variety of system-dependent parameters involved and their sometimes highly specific effects. Whether or not sorting of matching lipids around a membrane protein or peptide occurs in a particular system will depend on (i) the energy cost of mismatch of the transmembrane domain(s) with the bilayer, (ii) the energy cost of lipid sorting, which is due to a loss of entropy, and (iii) the energy cost of relieving mismatch in other ways, for example, by lipid acyl chain stretching, tilting of transmembrane segments, local backbone deformations, or peptide aggregation (reviewed in ref 30). Parameters that determine these energy costs include the extent of mismatch between the protein and the lipids, the length difference between the lipids, the mixing behavior of the pure lipids, and specific properties of the proteins or peptides. The lipid compositions investigated in the present study closely resemble those previously used in fluorescence studies, in which some preferential interactions were in fact observed $(5,7,8,31)$. However, the properties of the peptides and proteins used in the various studies are very different, and it is important to note that even small changes in amino acid sequence of a transmembrane peptide can result in very different behavior of a peptide/lipid system. This can be illustrated, for example, by the differences in mismatch responses in single lipid systems containing WALP peptides as compared to KALP peptides, which are analogous but are flanked by lysine residues instead of tryptophans (for review see ref 32 ).

A parameter that may be of special importance in determining the nature of the interactions at the protein/lipid interface, and hence in determining whether molecular sorting can occur, is the cross-sectional diameter of the transmembrane part of membrane proteins. Although WALP peptides resemble the transmembrane helices of large, multispanning $\alpha$-helical membrane proteins, they differ from those proteins in two important aspects. First, in multispanning proteins a larger number of lipids is influenced by one protein and of each surrounding lipid a slightly larger area is exposed to the protein. Second, multispanning proteins are relatively rigid entities within the bilayer due to intramolecular helix-helix interactions. It is therefore likely that the large, and relatively rigid wall of multispanning membrane proteins is more suited for stable interaction with a matching lipid than the much smaller and more dynamical outer surface of a single transmembrane peptide. This hypothesis would be in agreement with Monte Carlo simulations, which suggest that the influence of membrane proteins on the surrounding lipids increases with the cross-sectional diameter of the protein (33), and with recent experimental data, which suggest that effects of transmembrane peptides on bilayer thickness are dependent on their size (34). It is also supported by recent results describing effects of transmembrane peptides and proteins on flip-flop of phospholipids in bilayers (35). In pure lipid bilayers flip-flop is extremely slow, but incorporation of WALP or other transmembrane peptides, including natural ones, causes a remarkably increased rate of lipid flip-flop. Interestingly, increasing the size of the protein or protein oligomeric complex results in a gradual, decreased ability of the protein to cause lipid flipflop, clearly demonstrating that the size of the membranespanning part of a protein influences the dynamics of lipids at the protein-lipid interface. Also previous ESR studies on boundary lipids can be taken as support of the hypothesis that larger membrane proteins allow a more stable interaction with surrounding lipids. In these studies boundary lipids are generally readily identified in large multispanning proteins, but they are often not observed for single-span peptides, such as the WALP peptides (12). Together, these results imply that within biological membranes the size and rigidity of the transmembrane domains of membrane proteins are major determinants for the interaction with the membrane lipids. We propose that in general the large and relatively rigid outer surface of multispanning membrane proteins facilitates preferential sorting of matching lipids more so than the much smaller and more dynamical outer surface of single-transmembrane peptides.

\section{ACKNOWLEDGMENT}

J.A.K. thanks Maria Maddalena Sperotto for helpful discussions.

\section{REFERENCES}

1. Sperotto, M. M., and Mouritsen, O. G. (1993) Lipid enrichment and selectivity of integral membrane proteins in two-component lipid bilayers, Eur. Biophys. J. 22, 323-328.

2. Dumas, F., Sperotto, M. M., Lebrun, M. C., Tocanne, J. F., and Mouritsen, O. G. (1997) Molecular sorting of lipids by bacteriorhodopsin in dilauroylphosphatidylcholine/distearoylphosphatidylcholine lipid bilayers, Biophys. J. 73, 1940-1953.

3. Gil, T., Ipsen, J. H., Mouritsen, O. G., Sabra, M. C., Sperotto, M. M., and Zuckermann, M. J. (1998) Theoretical analysis of protein organization in lipid membranes, Biochim. Biophys. Acta 1376, $245-266$.

4. Dumas, F., Lebrun, M. C., and Tocanne, J. F. (1999) Is the protein/ lipid hydrophobic matching principle relevant to membrane organization and functions? FEBS Lett. 458, 271-277.

5. O'Keeffe, A. H., East, J. M., and Lee, A. G. (2000) Selectivity in lipid binding to the bacterial outer membrane protein $\mathrm{OmpF}$, Biophys. J. 79, 2066-2074.

6. Lehtonen, J. Y. A., and Kinnunen, P. K. J. (1997) Evidence for phospholipid microdomain formation in liquid crystalline liposomes reconstituted with Escherichia coli lactose permease, Biophys. J. 72, 1247-1257.

7. Fernandes, F., Loura, L. M. S., Prieto, M., Koehorst, R., Spruijt, R. B., and Hemminga, M. A. (2003) Dependence of M13 major coat protein oligomerization and lateral segregation on bilayer composition, Biophys. J. 85, 2430-2441.

8. Williamson, I. M., Alvis, S. J., East, J. M., and Lee, A. G. (2002) Interactions of phospholipids with the potassium channel KcsA, Biophys. J. 83, 2026-2038.

9. London, E., and Feigenson, G. W. (1981) Fluorescence quenching in model membranes. 2. Determination of local lipid environment of the calcium adenosinetriphosphatase from sarcoplasmic reticulum, Biochemistry 20, 1939-1948.

10. East, J. M., and Lee, A. G. (1982) Lipid selectivity of the calcium and magnesium ion dependent adenosinetriphosphatase, studied with fluorescence quenching by a brominated phospholipid, Biochemistry 21, 4144-4151.

11. Ryba, N. J., and Marsh, D. (1992) Protein rotational diffusion and lipid/protein interactions in recombinants of bovine rhodopsin with saturated diacylphosphatidylcholines of different chain lengths studied by conventional and saturation-transfer electron spin resonance, Biochemistry 31, 7511-7518.

12. De Planque, M. R., Greathouse, D. V., Koeppe, R. E., II, Schafer, H., Marsh, D., and Killian, J. A. (1998) Influence of lipid/peptide hydrophobic mismatch on the thickness of diacylphosphatidylcholine bilayers. A ${ }^{2} \mathrm{H}$ NMR and ESR study using designed 
transmembrane alpha-helical peptides and gramicidin A, Biochemistry 37, 9333-9345.

13. De Planque, M. R. R., Demmers, J. A. A., Bonev, B. B., Koeppe, R. E., II Greathouse, D. V., Separovic, F., Watts, A., and Killian J. A. (2003) Interfacial anchor properties of tryptophan residues in transmembrane peptides can dominate over hydrophobic matching effects in peptide-lipid interactions, Biochemistry 42, 5341-5348.

14. Demmers, J. A. A., Haverkamp, J., Heck, A. J. R., Koeppe II, R. E., and Killian, J. A. (2000) Electrospray ionization mass spectrometry as a tool to analyze hydrogen/deuterium exchange kinetics of transmembrane peptides in lipid bilayers, Proc. Natl. Acad. Sci. U.S.A. 97, 3189-3194.

15. Rinia, H. A., Kik, R. A., Demel, R. A., Snel, M. M., Killian, J. A., van der Eerden, J. P., and de Kruijff, B. (2000) Visualization of highly ordered striated domains induced by transmembrane peptides in supported phosphatidylcholine bilayers. Biochemistry $39,5852-5858$.

16. Kol, M. A., de Kroon, A. I., Rijkers, D. T. S., Killian, J. A., and de Kruijff, B. (2001) Membrane-spanning peptides induce phospholipid flop: a model for phospholipid translocation across the inner membrane of E. coli, Biochemistry 40, 10500-10506.

17. Van Duyl, B. Y., Rijkers, D. T. S., de Kruijff, B., and Killian, J. A. (2002) Influence of hydrophobic mismatch and palmitoylation on the association of transmembrane $\alpha$-helical peptides with detergent-resistant membranes, FEBS Lett. 523, 79-84.

18. De Planque, M. R. R., Kruijtzer, J. A. W., Liskamp, R. M. J., Marsh, D., Greathouse, D. V., Koeppe II, R. E., de Kruijff, B., and Killian, J. A. (1999) Different membrane anchoring positions of tryptophan and lysine in synthetic transmembrane alpha-helical peptides, J. Biol. Chem. 274, 20839-20846.

19. Bligh, E. G., and Dyer, W. J. (1959) A rapid method of total lipid extraction and purification, Can. J. Biochem. Physiol. 37, 911917.

20. Schägger, H., and von Jagow, G. (1987) Tricine-sodium dodecyl sulfate-polyacrylamide gel electrophoresis for the separation of proteins in the range from 1 to $100 \mathrm{kDa}$, Anal. Biochem. 166, 368-379.

21. Bollag, D. M., and Edelstein, S. J. (1991) in Protein Methods, Wiley-Liss, New York.

22. Brunner, J. (1993) New photolabeling and crosslinking methods, Annu. Rev. Biochem. 62, 483-514.

23. Weber, T., and Brunner, J. (1995) 2-(Tributylstannyl)-4-[3-(trifluoromethyl)-3H-diazirin-3-yl]benzyl alcohol: a building block for photolabeling and crosslinking reagents of very high specific radioactivity, J. Am. Chem. Soc. 117, 3084-3095.
24. Von Ballmoos, C., Appoldt, Y., Brunner, J., Granier, T., Vasella, A., and Dimroth, P. (2002) Membrane topography of the coupling ion binding site in $\mathrm{Na}^{+}$-translocating $\mathrm{F}_{1} \mathrm{~F}_{\mathrm{o}}$ ATP synthase, J. Biol. Chem. 277, 3504-3510.

25. De Planque, M. R. R., Goormaghtigh, E., Greathouse, D. V., Koeppe, R. E., II, Kruijtzer, J. A. W., Liskamp, R. M. J., de Kruijff, B., and Killian, J. A. (2001) Sensitivity of single membrane-spanning alpha-helical peptides to hydrophobic mismatch with a lipid bilayer: Effects on backbone structure, orientation, and extent of membrane incorporation, Biochemistry 40, 5000-5010.

26. Martoglio, B., Hofmann, M. W., Brunner, J., and Dobberstein, B. (1995) The protein-conducting channel in the membrane of the endoplasmic reticulum is open laterally toward the lipid bilayer, Cell 81, 207-214.

27. Brunner, J. (1996) Use of photo-crosslinkers in cell biology, Trends Cell Biol. 6, 154-157.

28. Horowitz, A. D. (1995) Exclusion of SP-C, but not SP-B, by gel phase palmitoyl lipids, Chem. Phys. Lipids 76, 27-39.

29. Mall, S., Broadbridge, R., Sharma, R. P., East, J. M., and Lee, A. G. (2001) Self-association of model transmembrane alpha-helices is modulated by lipid structure, Biochemistry 40, 12379-12386.

30. De Planque, M. R. R.; Killian J. A. (2003) Protein-lipid interactions studied with designed transmembrane peptides: role of hydrophobic matching and interfacial anchoring, Mol. Membr. Biol. 20, 271-284.

31. Webb, R. J., East, J. M., Sharma, R. P., and Lee, A. G. (1998) Hydrophobic mismatch and the incorporation of peptides into lipid bilayers: a possible mechanism for retention in the Golgi, Biochemistry 37, 673-379.

32. Killian, J. A. (2003) Synthetic peptides as models for intrinsic membrane proteins, FEBS Lett. 555, 134-138.

33. Sperotto, M. M., and Mouritsen, O. G. (1991) Monte Carlo simulation studies of lipid order parameter profiles near integral membrane proteins, Biophys. J. 59, 261-270.

34. Weiss, T. M., van der Wel, P. C. A., Killian, J. A., Koeppe, R. E., II, and Huang, H. (2003) Hydrophobic mismatch between helices and lipid bilayers, Biophys. J. 84, 379-385.

35. Kol, M. A., van Dalen, A., de Kroon, A. I. P. M., and de Kruijff, B. (2003) Phospholipid flop induced by transmembrane peptides in model membranes is modulated by lipid composition, J. Biol. Chem. 278, 24586-24593.

BI049899D 